\title{
Influence of strain relaxation on magnetotransport properties of epitaxial $\mathrm{La}_{0.7} \mathrm{Ca}_{0.3} \mathrm{MnO}_{3}$ films
}

\author{
P K Siwach ${ }^{1,3}$, H K Singh ${ }^{2}$ and O N Srivastava ${ }^{1}$ \\ ${ }^{1}$ Physics Department, Banaras Hindu University, Varanasi-221005, India \\ ${ }^{2}$ National Physical Laboratory, Dr K S Krishnan Road, New Delhi-110012, India \\ E-mail: pksiwach@yahoo.com and pk.siwach@jiit.ac.in
}

Received 8 June 2006, in final form 22 September 2006

Published 13 October 2006

Online at stacks.iop.org/JPhysCM/18/9783

\begin{abstract}
In this paper, we study the effect of strain relaxation on the magnetotransport properties of $\mathrm{La}_{0.7} \mathrm{Ca}_{0.3} \mathrm{MnO}_{3}$ (LCMO) epitaxial films (200 nm thick), which were deposited by pulsed laser deposition technique under identical conditions. All the films are epitaxial and have a cubic unit cell. The amount of strain relaxation has been varied by taking three different single crystal substrates of $\mathrm{SrTiO}_{3}, \mathrm{LaAlO}_{3}$ and $\mathrm{MgO}$. It has been found that for thicker films the strain becomes relaxed and produces a variable amount of disorder depending on the strength of strain relaxation. The magnitude of lattice relaxation has been found to be $0.384 \%, 3.057 \%$ and $6.411 \%$ for film deposited on $\mathrm{SrTiO}_{3}$, $\mathrm{LaAlO}_{3}$ and $\mathrm{MgO}$ respectively. The films on $\mathrm{LaAlO}_{3}$ and $\mathrm{SrTiO}_{3}$ show higher $T_{\mathrm{IM}}$ (insulator-metal transition temperature) of $\sim 243$ and $217 \mathrm{~K}$ respectively as compared to $T_{\mathrm{IM}}$ of $\sim 191 \mathrm{~K}$ for the film on $\mathrm{MgO}$. Similarly the Curie temperature $T_{\mathrm{C}}$ of the films on $\mathrm{SrTiO}_{3}$ and $\mathrm{LaAlO}_{3}$ is sharper and has values of $\sim 245$ and $220 \mathrm{~K}$ respectively, whereas the $T_{\mathrm{C}}$ of the film on $\mathrm{MgO}$ is $\sim 186 \mathrm{~K}$. A higher degree of relaxation creates more defects and hence the $T_{\mathrm{IM}}\left(T_{\mathrm{C}}\right)$ of the film on $\mathrm{MgO}$ is significantly lower than those of $\mathrm{SrTiO}_{3}$ and $\mathrm{LaAlO}_{3}$. We have adopted a different approach to correlate the effect of strain relaxation on the magnetotransport properties of LCMO films by evaluating the resistivity variation through Mott's variable range hopping (VRH) model. The variable presence of disorder in these thick films due to lattice relaxation, which have been analysed through Mott's VRH model, provides strong additional evidence that the strength of lattice relaxation produces disorder dominantly by an increase in density of defects such as stacking faults and dislocations, which affect the magnetotransport properties of thick epitaxial $\mathrm{La}_{0.7} \mathrm{Ca}_{0.3} \mathrm{MnO}_{3}$ films.
\end{abstract}

3 Author to whom any correspondence should be addressed. Present address: Jaypee Institute of Information Technology (Deemed University), A-10, Sector-62, Noida-201307, UP, India. 


\section{Introduction}

The discovery of the colossal magnetoresistance (CMR) effect in epitaxial manganite thin films has renewed interest in these materials for technological usefulness such as potential sensor and magnetic recording applications as well as the need to understand the mechanisms underlying their behaviour [1-3]. In epitaxial films, biaxial strain has been reported to have very strong effect on the changes in $T_{\mathrm{C}}$ and insulator-metal transition temperature $\left(T_{\mathrm{IM}}\right)$ [4-23]. Several publications have reported that both $T_{\mathrm{C}}$ and $T_{\mathrm{IM}}$ can vary with film thickness [14-23]. In most cases, these changes are interpreted in terms of substrate-induced strain, which relaxes with increase in thickness [18-23]. The lattice mismatch $\delta$ along the interface is defined by $\delta=\left(a_{p \text { substrate }}-a_{p \text { bulk }}\right) / a_{p \text { substrate }}$. When the film is grown on a substrate whose lattice parameter is smaller or larger than that of the bulk material, the epitaxial strain is expected to be compressive (the cell is elongated along the growth direction and compressed in the film's plane) or tensile (the cell is elongated in the film's plane and compressed along the out-plane growth direction), respectively. Compressive strain usually reduces the resistivity and shifts $T_{\mathrm{C}}$ towards higher temperature. These effects have been confirmed in $\mathrm{La}_{0.7} \mathrm{Ca}_{0.3} \mathrm{MnO}_{3}$ films [6, 7] and $\mathrm{La}_{0.7} \mathrm{Sr}_{0.3} \mathrm{MnO}_{3}$ films [8-12] grown on various substrates.

The observed strain effect is usually interpreted qualitatively within the double-exchange model, since the hopping matrix element $t$ could be altered by epitaxial strain. Thus a compressive/tensile strain induces an increase/decrease in $T_{\mathrm{IM}}$ by an increase/decrease in electron transfer due to the compressed/expanded $\mathrm{Mn}-\mathrm{O}$ bond lengths [6, 15]. However, recent detailed studies show that compressive strain does not always lead to enhancement of $T_{\mathrm{C}}$ [13], while the cationic vacancies due to the oxygen annealing significantly enhance the $T_{\mathrm{C}}$ values to become much higher than the corresponding bulk value [5, 14]. In most cases, tensile strain suppresses ferromagnetism and reduces $T_{\mathrm{C}}$ in manganite films. But some anomalous results have also been reported, showing that $T_{\mathrm{C}}$ is enhanced by tensile strain $[18,24,25]$. In a recent study Zhang et al [18] showed that a biaxial tensile strain can also lead to an enhancement of $T_{\mathrm{IM}}$ in $\mathrm{La}_{1-x} \mathrm{Ba}_{x} \mathrm{MnO}_{3}$ films due to orbital rearrangement. Nevertheless, the $T_{\mathrm{IM}}$ enhancement mechanism based on orbital rearrangements has not been expected to cause an increase of $T_{\mathrm{IM}}$ in the $\mathrm{La}_{1-x} \mathrm{Ca}_{x} \mathrm{MnO}_{3}$ system [19]. It has been also proposed that Jahn-Teller electron-phonon coupling plays an important role in the strain effect on $T_{\mathrm{C}}$ [26]. Most interestingly, there are reports of multiple phase segregation in strained epitaxial films [17]. The ferromagnetic coupling within the metallic regions accounts for the changes of $T_{\mathrm{C}}$ and conductivity.

These strain effects have been evaluated by the dependence of properties on the thickness and lattice matching between the films and substrates. Although consistent behaviours have been reported concerning the thickness dependence of $T_{\mathrm{C}}$ of the manganite films [19, 27, 28], disagreement exists concerning the origin of the observed phenomena. Some investigators have argued that the difference in oxygen content is the most important factor responsible for the $T_{\mathrm{C}}$ variation in manganites and that strain has less effect $[12,28]$, while others have claimed that a change in structure, which is strongly coupled with the electronic system, must account for the origin of the behaviours observed $[19,29]$. Thus, the strain effect in manganite films has not become completely intelligible.

In the present work we have synthesized and studied the magnetotransport properties of $\mathrm{La}_{0.7} \mathrm{Ca}_{0.3} \mathrm{MnO}_{3}$ epitaxial films deposited on $\mathrm{LaAlO}_{3}, \mathrm{SrTiO}_{3}$ and $\mathrm{MgO}$ single crystal substrates by pulsed laser deposition. It is known that lattice strain due to film-substrate mismatch affects the properties for thin films $(<100 \mathrm{~nm})$ and it influences the magnetotransport properties $\left(T_{\mathrm{IM}}, T_{\mathrm{C}}\right.$ and $\left.\mathrm{MR}\right)$ significantly. However, for thicker films $(>100 \mathrm{~nm})$, the lattice strain gets relaxed and produces various kinds of imperfections such as stacking faults and dislocations. In strain-relaxed epitaxial thin films of $\mathrm{La}_{0.7} \mathrm{Ca}_{0.3} \mathrm{MnO}_{3}$, significant low-field $\mathrm{MR}$ 
has been observed and this is supposed to be closely related to the degree of lattice relaxation and hence the density of defects that disrupt the long-range order [30, 34]. In the present work the possible correlation between the degree of strain relaxation and the various physical properties such as $T_{\mathrm{IM}}, T_{\mathrm{C}}$ and low-field MR has been investigated for relatively thicker films ( $200 \mathrm{~nm}$ ) of $\mathrm{La}_{0.7} \mathrm{Ca}_{0.3} \mathrm{MnO}_{3}$ deposited by pulsed laser deposition.

\section{Experimental details}

\subsection{Preparation of ceramic targets}

For the preparation of a film by pulsed laser deposition (PLD), first a stoichiometric ceramic target has to be synthesized. The $\mathrm{La}_{0.7} \mathrm{Ca}_{0.3} \mathrm{MnO}_{3}$ target was prepared by standard solid-state reaction of appropriate mixtures of $3 \mathrm{~N}$ pure $\mathrm{La}_{2} \mathrm{O}_{3}, \mathrm{CaO}$ and $\mathrm{MnCO}_{3}$. The powders were mixed thoroughly and calcined at $900^{\circ} \mathrm{C}$ for $12 \mathrm{~h}$ in air. This process of mixing and calcinations was repeated several times; each time the temperature was raised by 50 to $100^{\circ} \mathrm{C}$ up to $1100{ }^{\circ} \mathrm{C}$. After that the powder was pressed into a round pellet (target). The diameter of the targets is $20 \mathrm{~mm}$, while the thickness is about $3 \mathrm{~mm}$. The target was then sintered in air at $1300^{\circ} \mathrm{C}$ for $36 \mathrm{~h}$, and finally annealed in flowing oxygen at $1000^{\circ} \mathrm{C}$ for $12 \mathrm{~h}$. This produces very dense and hard targets so that particulate contamination of the laser-deposited films is reduced as much as possible.

\subsection{Deposition of films}

The procedure we have used to grow thin manganite films is as follows. Prior to deposition, the substrates (for present study $\mathrm{LaAlO}_{3}, \mathrm{SrTiO}_{3}$ and $\mathrm{MgO}$; all in (100) orientation) were cleaned successively twice in acetone and isopropanol to remove dust or any other greasy contaminations. All three substrates were then glued side-by-side on the heater with silver paste to ensure good thermal contact and to have identical synthesis condition. The deposition chamber was evacuated down to a background pressure of $\sim 10^{-5}$ mbar or less. Then the vacuum pump was switched to a lower speed and the oxygen inlet, controlled manually by a needle valve, was opened to reach a constant, dynamic oxygen pressure (i.e. the deposition pressure) of $200-400$ mbars. A typical temperature for film deposition is $700-800^{\circ} \mathrm{C}$, the temperature being monitored by a thermocouple attached to the heater. In the meantime, the laser was switched on and brought to its maximum output power. After stabilization of pressure, temperature and laser power for about $15 \mathrm{~min}$, the target was ablated for several minutes with the shutter closed in front of the heater. This is to remove all possible contaminations from the target surface without depositing material onto the substrate. The laser beam $(\lambda=248 \mathrm{~nm})$ is focused on a target with an energy density of $\sim 2 \mathrm{~J} \mathrm{~cm}^{-2}$ in a spot of $3-4 \mathrm{~mm}^{2}$ with repetition rate (pulse frequency) of $2 \mathrm{~Hz}$; the actual focus is set just in front of the target to minimize interaction between ejected ions and the laser beam. During the entire ablation process the deposition chamber was kept at a dynamic oxygen pressure of 200-400 mbar. The heater, carrying the substrate, is held at a distance of $\sim 4 \mathrm{~cm}$ from the target. The films presented in this study were all deposited in on-axis geometry, i.e. perpendicular incidence of plume to substrate. The film thickness was monitored by the number of ablating laser pulses during deposition. The typical growth rate for the manganites at the parameters used is around $10 \mathrm{~nm}$ per minute. After the desired deposition time (typically around $20 \mathrm{~min}$ yielding a film thickness of around $200 \mathrm{~nm}$ ), the laser was switched off, and the sample was kept in an oxygen atmosphere (usually $1 \mathrm{bar}$ ) at the deposition temperature (for $\geqslant 1 \mathrm{~h}$ ) and then slowly cooled (typically $5^{\circ} \mathrm{min}^{-1}$ ) 


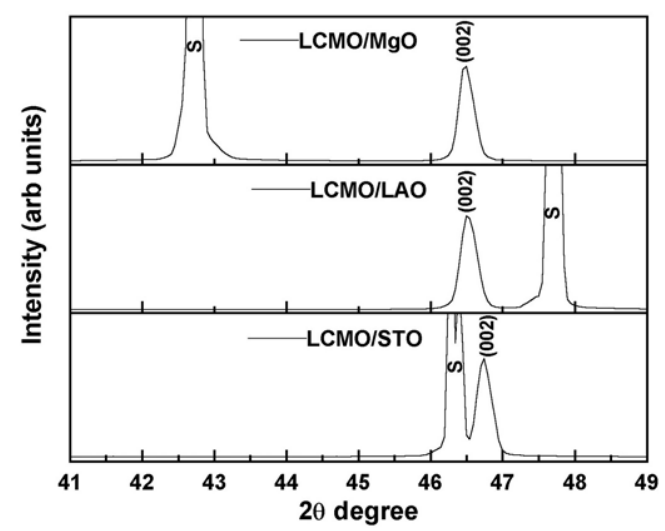

Figure 1. XRD pattern of pulsed laser deposited epitaxial films of $\mathrm{La}_{0.7} \mathrm{Ca}_{0.3} \mathrm{MnO}_{3}$ (LCMO) on STO, LAO and $\mathrm{MgO}$ substrates.

down to room temperature. After that the films were post-annealed in flowing oxygen at $1000^{\circ} \mathrm{C}$ for $8 \mathrm{~h}$ to ensure full oxygen content.

\subsection{Structural and magnetotransport characterizations}

The crystal structure and orientation of all films were characterized by $\mathrm{x}$-ray diffraction using $\mathrm{Cu} \mathrm{K} \alpha$ radiation $(\lambda=1.54106 \AA)$ at room temperature in the $2 \theta$ range $20^{\circ}-80^{\circ}$. X-ray rocking curve analysis was done to check the crystallinity of the films. The magnetization measurements were performed in a vibrating sample magnetometer at an applied magnetic field of $153 \mathrm{Oe}$ in the range $77-300 \mathrm{~K}$. The electrical resistance was measured in the range 77-300 K by the four-probe technique. The MR was calculated by using the resistance data with and without applied field $\left(H_{\mathrm{dc}}=1.0 \mathrm{~T}\right)$, which was applied parallel to film plane.

\subsection{Results and discussions}

2.4.1. Structure analysis. The XRD patterns of $\mathrm{La}_{0.7} \mathrm{Ca}_{0.3} \mathrm{MnO}_{3}$ (LCMO) films fabricated on $\mathrm{SrTiO}_{3}(001), \mathrm{LaAlO}_{3}$ were found to match with the $(00 l)$ reflections expected from the bulk. The pattern confirms that the films are indeed single phase without any impurity and grown epitaxially on the $c$-axis, as shown in figure 1. In order to study the effect of the substrate on the structure of the deposited films, the (002) peaks in the XRD pattern were analysed.

The out-of-plane lattice parameters of the LCMO thin films deposited on STO, LAO and $\mathrm{MgO}$ single crystal substrates were measured to be $3.878,3.889$ and $3.899 \AA$ respectively and that for the bulk LCMO target is $3.92 \AA$. The in-plane lattice parameters of LCMO thin films were obtained from an asymmetric XRD scan of the (311) reflections and they are 3.890, 3.911 and $3.912 \AA$ respectively on STO, LAO and MgO substrates. Thus there is no appreciable difference between the in-plane and out-of-plane lattice parameters. The near equality of in-plane and out-of-plane lattice parameters is due to the fact that the thickness of these films $(\sim 200 \mathrm{~nm})$ is much larger than the critical thickness up to which the biaxial strain originating due to the difference between the lattice parameters of the substrate and LCMO can be effective. Since the film thickness is larger the lattice strain will get relaxed. At film thicknesses larger than the critical thickness, the lattice relaxation, defined as $L=$ $\left[\left(a_{\text {film }}-a_{\text {substrate }}\right) \times 100\right] / a_{\text {substrate }}$, will play the dominant role. In fact it has been shown that at larger thickness such as $\sim 200 \mathrm{~nm}$, the lattice strain relaxes, leading to the generation of a 


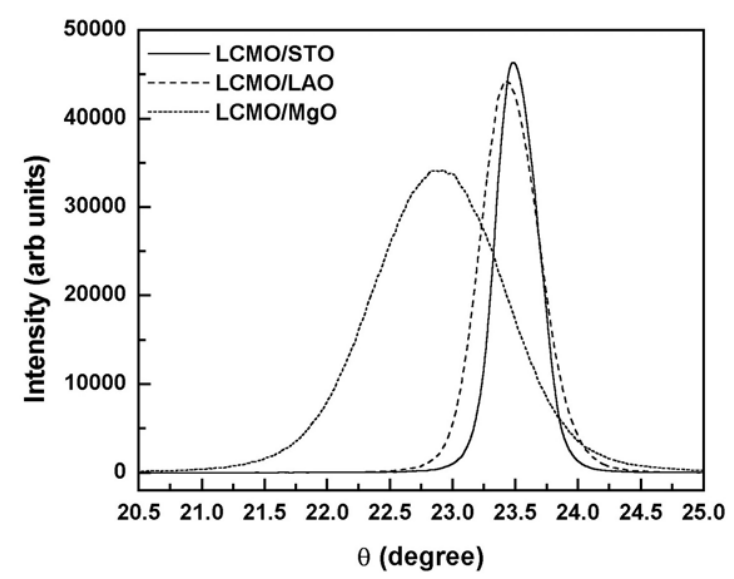

Figure 2. $\omega$ scan of the (002) reflection for LCMO films on STO, LAO and MgO.

variety of defects [30]. The epitaxial nature of the films has also been confirmed by the rocking curve analysis of the films, and rocking curves corresponding to the (002) reflection are shown in figure 2. As seen in the figure, in the case of the LCMO/STO film the intensity is highest and it decreases for $\mathrm{LCMO} / \mathrm{LAO}$ and $\mathrm{LCMO} / \mathrm{MgO}$ films in that order. Similarly the FWHM is observed to be the minimum for the LCMO/STO film and increases for LCMO/LAO and $\mathrm{LCMO} / \mathrm{MgO}$ in that order. The FWHM for these films is $0.39^{\circ}, 0.52^{\circ}$ and $1.24^{\circ}$ respectively.

The broadening observed above may be due to one or more of the following factors: (i) the mosaic spread, (ii) reduction of the long-range order in the lattice, and (iii) increased static disorder due to imperfect growth. The long-range order can be disrupted by the increased density of the stacking faults and tilting out of the in-plane grains. Static disorders may lead to the effects such as electron localization. In the present case the magnitude of lattice relaxation has been found to be $0.384 \%, 3.057 \%$ and $6.411 \%$ for films deposited on STO, LAO and $\mathrm{MgO}$ single crystal substrates respectively [30]. Obviously as the difference between the lattice parameters of the LCMO film and substrates increases the magnitude of lattice relaxation also increases. At the same time the FWHM of the rocking curves is found to increase with the lattice relaxation. Increasing FWHM as evidenced by the broadened rocking curves with increasing lattice relaxation shows that defects are being generated as a consequence of this relaxation. As mentioned earlier, the lattice relaxation indeed gives rise to extrinsic distortions/defects such as dislocations, grain-boundaries, stacking faults, and cationic vacancies. The lattice relaxation may not appreciably affect the more fundamental features such as the magnetic exchange interactions, but it may affect Jahn-Teller distortions and hence the electron lattice coupling. Thus the effects of lattice relaxation will get reflected in the magnetotransport characteristics [7, 9, 18, 19, 22, 23, 28].

2.4.2. Magnetotransport properties. The paramagnetic-ferromagnetic (PM-FM) phase transition was studied by measuring the magnetization $(M)$ in the temperature range 300$77 \mathrm{~K}$ and the $M-T$ data are plotted in figure 3(a). The PM-FM transition temperature or the Curie temperature $\left(T_{\mathrm{C}}\right)$ was determined from the maxima in the magnitude of the $\mathrm{d} M / \mathrm{d} T$. The observed $T_{\mathrm{C}}$ values are $\sim 244,218$ and $186 \mathrm{~K}$ respectively for LCMO films deposited on STO, LAO and MgO. The $T_{\mathrm{C}}$ of the bulk target has been measured to be $\sim 245 \mathrm{~K}$ and the corresponding $M-T$ data are plotted in figure $3(\mathrm{~b})$. The $T_{\mathrm{C}}$ of the LCMO bulk target and the LCMO thin film on STO substrate are nearly equal while those of the LCMO films on LAO 

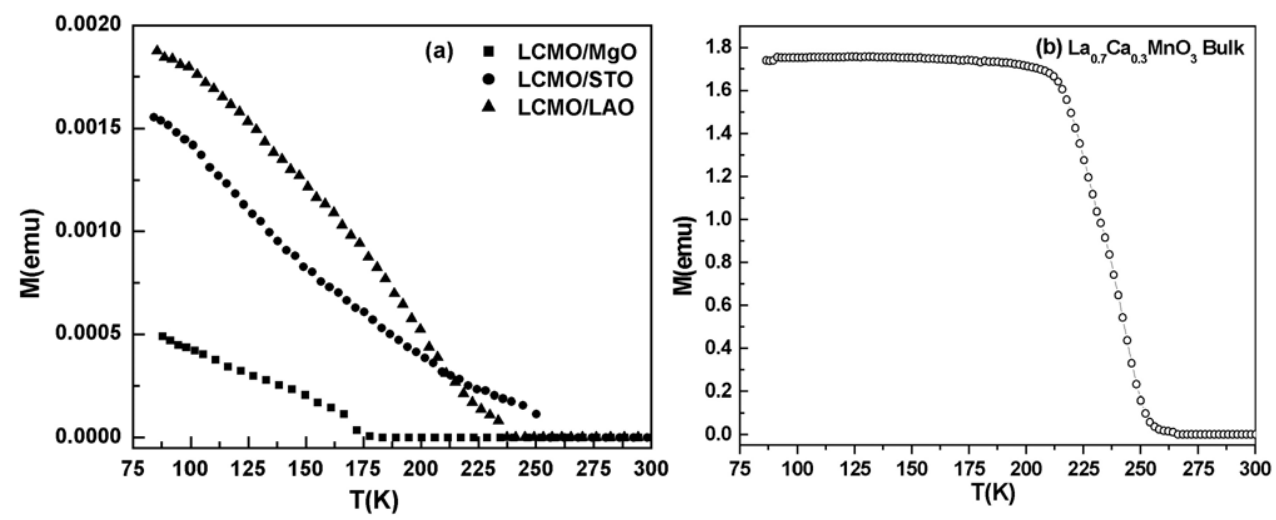

Figure 3. Temperature dependence of the magnetization for (a) LCMO films on STO, LAO and $\mathrm{MgO}$ substrates and (b) bulk $\mathrm{La}_{0.7} \mathrm{Ca}_{0.3} \mathrm{MnO}_{3}$.

Table 1. The various characteristics parameters of epitaxial films of $\mathrm{La}_{0.7} \mathrm{Ca}_{0.3} \mathrm{MnO}_{3}$ on $\mathrm{SrTiO}_{3}(001), \mathrm{LaAlO}_{3}(001)$ and $\mathrm{MgO}(001)$.

\begin{tabular}{llll}
\hline Characteristics & STO $(3.905 \AA)$ & LAO $(3.821 \AA)$ & MgO (4.216 $)$ \\
\hline Out of plane lattice constant $(\AA)$ & 3.878 & 3.889 & 3.899 \\
Lattice relaxation $L=\left(a_{\mathrm{F}}-a_{\mathrm{S}}\right) \times 100 / a_{\mathrm{S}}$ & -0.384 & 3.057 & -6.411 \\
$T_{\mathrm{C}}(\mathrm{K})$ & 245 & 220 & 186 \\
$T_{\mathrm{IM}}(\mathrm{K})$ & 243 & 217 & 191 \\
$\rho$ at $300 \mathrm{~K}$ and at $T_{\mathrm{IM}}(\mathrm{m} \Omega \mathrm{cm})$ & 36 & 31 & 38 \\
& 72 & 113 & 275 \\
$\rho\left(T_{\mathrm{IM}}\right) / \rho(300 \mathrm{~K})$ & 2 & 3.65 & 7.25 \\
$\mathrm{MR} \%(0.5 \mathrm{~T})\left(\right.$ At $T \sim T_{\mathrm{C}}, T_{\mathrm{IM}}$ and $\left.80 \mathrm{~K}\right)$ & $2.5,3.5,1.4$ & $7.1,11.2,0.3$ & $13.9,21,3.5$ \\
$\mathrm{MR} \%(1.0 \mathrm{~T})\left(\right.$ At $T \sim T_{\mathrm{C}}, T_{\mathrm{IM}}$ and $\left.80 \mathrm{~K}\right)$ & $9.1,12.5,2.1$ & $16.5,5.9,4.4$ & $25.7,38,4.7$ \\
$\mathrm{MR}_{\max }(0.5 \mathrm{~T}, 1.0 \mathrm{~T})$ & $6.9,17.6$ & $22.5,41$ & 26,44 \\
$T(\mathrm{MR}$ & 220,225 & 195,200 & 175,180 \\
$T_{0}(\mathrm{~K})$ & $1.242 \times 10^{7}$ & $2.251 \times 10^{7}$ & $3.830 \times 10^{7}$ \\
\hline
\end{tabular}

and $\mathrm{MgO}$ substrates are smaller than that of the bulk LCMO target. In fact, the $M-T$ data show that the transitions in the LCMO thin films on LAO and $\mathrm{MgO}$ substrates are not as pronounced as in case of the LCMO bulk and thin film on STO. The broadness of the transition suggests either a percolative regime or a fluctuation regime having its origin in the competition between related interactions around $T_{\mathrm{C}}$. There are host of factors that can account for a $T_{\mathrm{C}}$ depression as observed in the LCMO thin films grown on LAO and $\mathrm{MgO}$ substrates. One of such possible factors is the biaxial lattice strain, but as the film thickness is around $\sim 200 \mathrm{~nm}$, the lattice strain is expected to be almost completely relaxed. Therefore the contribution from this factor will be negligibly small $[7,9,18,19,22,23,28]$. The second factor leading to $T_{\mathrm{C}}$ depression is oxygen deficiency, and it may well be one of the reasons leading to $T_{\mathrm{C}}$ depression in the present case, especially in the case of films deposited on $\mathrm{MgO}$ substrates. Another reason and perhaps the dominant one in the present study might well be the structural disorders originating due the lattice strain relaxation. In fact, as can be seen in table 1, in the case of LCMO film on STO where the magnitude of lattice relaxation is very small $(0.384 \%)$, the $T_{\mathrm{C}}$ of the film is nearly the same as that of the bulk.

However, as the magnitude of the lattice relaxation increases to $3.057 \%$ and $6.411 \%$ for LCMO films on $\mathrm{LAO}$ and $\mathrm{MgO}, T_{\mathrm{C}}$ decreases quite rapidly to 218 and $186 \mathrm{~K}$, respectively. 


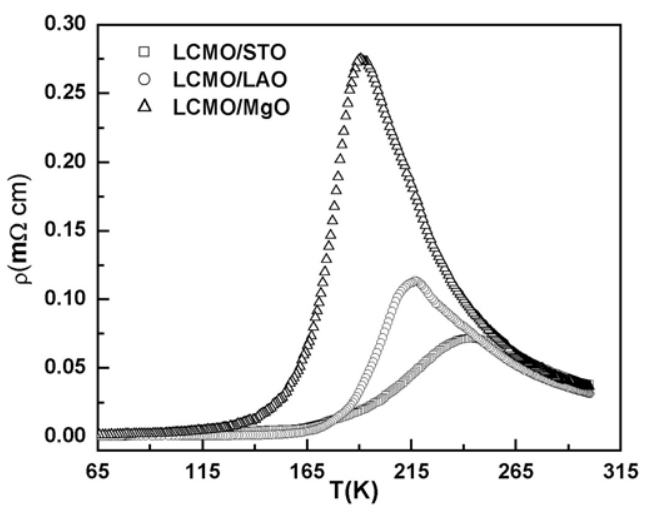

Figure 4. Typical temperature dependence of electrical resistivity for LCMO films on STO, LAO and $\mathrm{MgO}$.

The $T_{\mathrm{C}}$ depression can be accounted for as follows. As pointed out and discussed earlier, due to the difference between the lattice parameters of the substrate and film, lattice relaxation occurs. This may lead to the generation of various types of defects, such as mosaic spread and decrease in the long-range order due to increased density of stacking faults, in-plane tilting of grains, creation of oxygen vacancies, etc. It is expected that the density of such disorders would increase as the magnitude of the lattice relaxation increases. Induced disorders that disrupt the long-range order in the lattice, such as stacking faults, may produce modifications in the JahnTeller distortion of the $\mathrm{MnO}_{6}$ octahedra leading to electron localization effects and hence may increase the polaronic component to the transport in the paramagnetic phase. The larger value of the lattice relaxation in $\mathrm{LCMO} / \mathrm{MgO}$ film thus results in the higher density of defects such as stacking faults and dislocations. The increased density of these lattice defects may lead to larger distortion of the local lattice environment such as the $\mathrm{MnO}_{6}$ octahedra.

The temperature dependence of resistivity of all the LCMO thin films was measured between 300 and $77 \mathrm{~K}$ and the corresponding data are plotted in figure 4. At room temperature $(\sim 300 \mathrm{~K})$ the resistivity of LCMO films on STO, LAO and MgO is measured to be 36,31 and $38 \mathrm{~m} \Omega \mathrm{cm}$. Thus at room temperature all the films, irrespective of the substrate, have nearly the same resistivity value. However, as the temperature is reduced the resistivities are observed to increase and at a temperature well below room temperature an insulator-metal transition $\left(T_{\mathrm{IM}}\right)$ is observed. The $T_{\mathrm{IM}}$ of LCMO films on STO, LAO and $\mathrm{MgO}$ is found to be 243,217 and $191 \mathrm{~K}$ and the corresponding resistivities at $T_{\mathrm{IM}}$ are 72,113 and $275 \mathrm{~m} \Omega \mathrm{cm}$, respectively. Thus compared to the room-temperature values the resistivity of LCMO films on STO, LAO and $\mathrm{MgO}$ undergo a 2-fold, 3.65-fold and 7.25-fold increase while the $T_{\mathrm{IM}}$ for the LCMO film on $\mathrm{MgO}$ is higher than the $T_{\mathrm{C}}$. At $77 \mathrm{~K}$ the resistivity of all the films have been measured to be less than $2 \mathrm{~m} \Omega \mathrm{cm}$. Thus the observed variation of the resistivity of the LCMO films shows that increased lattice relaxation enhances the resistivity at $T_{\mathrm{IM}}$ while the room-temperature and the low-temperature resistivity remain nearly independent of the nature of the substrate and hence the lattice relaxation. The resistivity enhancement around $T_{\mathrm{IM}}$ and in the paramagnetic phase in the case of LCMO film having larger lattice relaxation suggests increased carrier localization.

2.4.3. Nature of transport. As outlined above, disorders are invariably present in the present films. The $\rho-T$ data above $T_{\mathrm{C}}$ were analysed in the framework of Mott's variable range hopping 

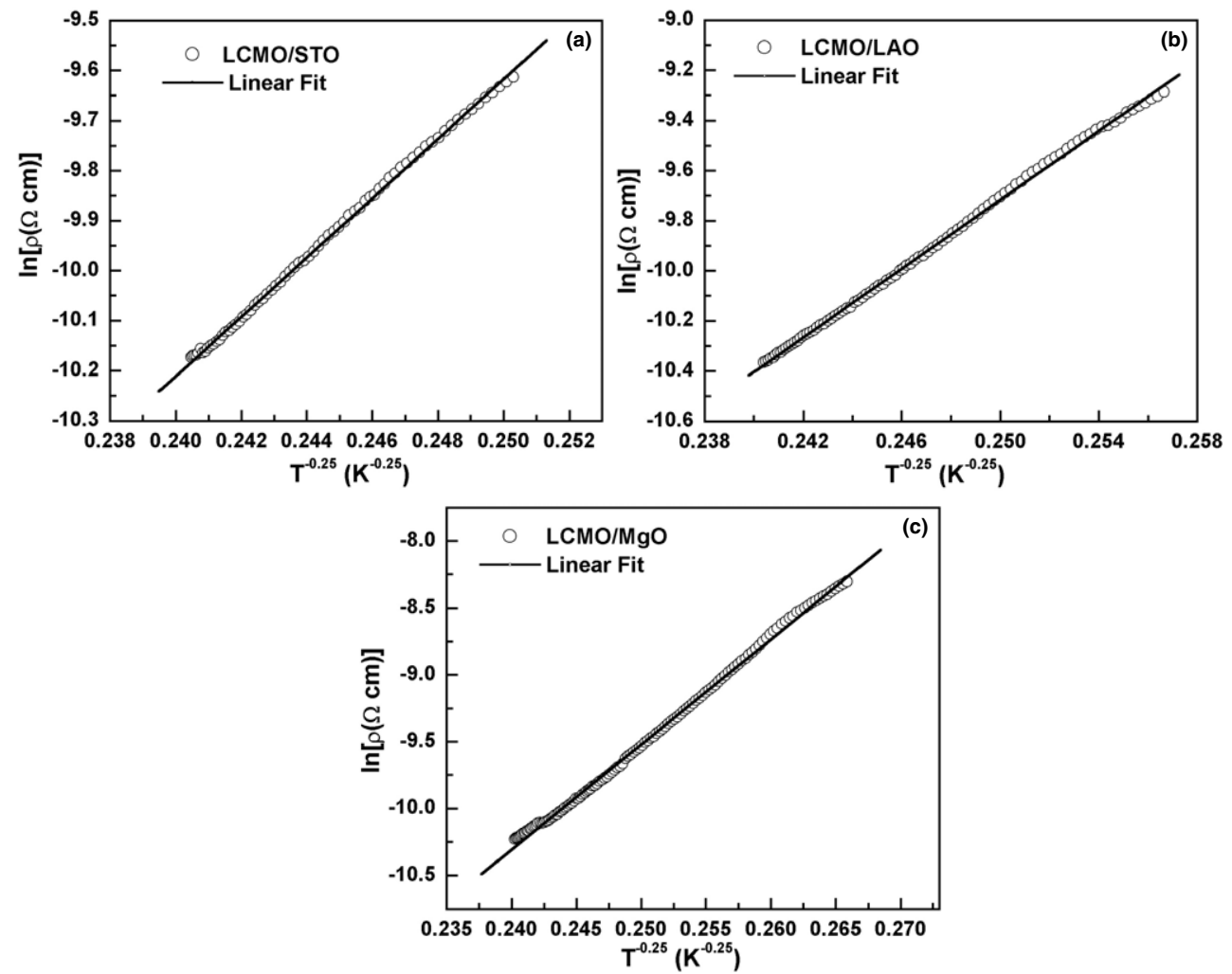

Figure 5. Variation of $\ln (\rho)$ with $T^{-0.25}$ for LCMO thin films on $\mathrm{SrTiO}_{3}, \mathrm{LaAlO}_{3}$ and $\mathrm{MgO}$ substrates. The open circles show the experimental data and the solid line is the best linear fit.

(VRH) model of polarons, which is given by [31]

$$
\rho(T)=\rho_{0} \exp \left(\frac{T_{0}}{T}\right)^{1 / 4}
$$

where $\rho_{0}$ is the residual resistivity and $T_{0}$ is the characteristic VRH temperature. It has been observed that in all LCMO films the data above $T_{\mathrm{C}}$ fit well to the above Mott's VRH equation. This is amply clear in the $\ln (\rho)-T^{-0.25}$ plots for the LCMO films as depicted in figures $5(\mathrm{a})-(\mathrm{c})$.

The value of the constant $\left(T_{0}\right)$ at room temperature was evaluated from the linear fit of the resistivity data and for LCMO films on STO, LAO and $\mathrm{MgO}$ the values of $T_{0}$ were found to be $1.24 \times 10^{7}, 2.25 \times 10^{7}$ and $3.83 \times 10^{7} \mathrm{~K}$, respectively. The value of $T_{0}$ obtained for our LCMO films on STO, LAO and MgO is in excellent agreement with the value reported by other workers [32-34]. Thus with increased lattice relaxation the characteristic VRH temperature $\left(T_{0}\right)$ also increases. Since the value of the parameter $T_{0}$ is a measure of the strength of the Jahn-Teller distortion and is inversely related to the extent of the localized states, the increasing value of $T_{0}$ suggests that increased lattice relaxation decreases the localization length which in turn reduces the average hopping distance.

The localization length $(1 / \alpha)$ was calculated using the modified formula proposed by Viret et al [32]. They have proposed that in case of manganites with $30 \%$ divalent cation doping at the rare earth site, the carrier localization above $T_{\mathrm{C}}$ is caused by a random potential of magnetic 
origin. This potential is due to the Hund's rule coupling $-J_{\mathrm{H}} \vec{S}_{i} \cdot \vec{S}_{j}$ between localized Mn t $2 \mathrm{~g}$ ion cores $(S=3 / 2)$ and spins of $\mathrm{e}_{\mathrm{g}}$ electrons in the conduction band. The modified formula for localization length $(1 / \alpha)$ in this model is given by

$$
\frac{1}{\alpha}=\left(\frac{171 U_{\mathrm{m}} V}{k_{\mathrm{B}} T_{0}}\right)^{1 / 3} \text {. }
$$

Here $U_{\mathrm{m}}=3 \frac{J_{\mathrm{H}}}{2}$ is the splitting between the spin-up and spin-down $\mathrm{e}_{\mathrm{g}}$ bands and its value has been found from optical spectra to be $2 \mathrm{eV}$ [35]. $V$ is the unit cell volume per $\mathrm{Mn}$ ion, $T_{0}$ is the characteristic VRH temperature and $k_{\mathrm{B}}$ is the Boltzmann constant. Substituting the values of $U_{\mathrm{m}}$ and $k_{\mathrm{B}}$, the above expression becomes

$$
\frac{1}{\alpha}=\left(\frac{3.965 \times 10^{6} V}{T_{0}}\right)^{1 / 3} .
$$

Thus the average nearest-neighbour hopping distance $(R)$ is given by

$$
R=\left(\frac{9}{8 \pi \alpha N(E) k_{\mathrm{B}} T}\right)^{1 / 4}
$$

where $N(E)$ is the density of available states in the random potential regime of Viret et al [35] and its value estimated by them for LCMO is $9 \times 10^{26} \mathrm{~m}^{-3} \mathrm{eV}^{-1}$. Using this value of $N(E)$ and the Boltzmann constant the above expression transform to

$$
R=\left(\frac{4.6112 \times 10^{-24}}{\alpha T}\right)^{1 / 4}
$$

Using the above expressions the value of the localization length $1 / \alpha$ has been calculated to be $2.65,2.18$ and $1.83 \AA$ for LCMO films on STO, LAO and MgO respectively. Similarly, the average hopping distance $R$ has been found to be 14.21, 13.53 and $12.98 \AA$ for LCMO films grown on STO, LAO and $\mathrm{MgO}$, respectively. From these data of localization length $(1 / \alpha)$ and average hopping distance $(R)$, it becomes evident that as the lattice relaxation increases in the sequence $\mathrm{STO}(-0.384) \rightarrow \operatorname{LAO}(3.057) \rightarrow \mathrm{MgO}(-6.411)$ the localization length sequentially decreases, that is, the carriers becomes more and more localized, resulting in a decrease in the average hopping distance. This also suggests that the lattice relaxation indeed leads to the generation of defects such as stacking faults and dislocations. The decrease in the localization length $(1 / \alpha)$ and average hopping distance $(R)$ is quite significant: $4.8 \%$ and $8.7 \%$ as compared to the values for the LCMO thin film on STO.

The low-field magnetoresistance (LFMR) of the LCMO thin films grown on different substrates was measured in the temperature range 300-77 K and at magnetic fields $H_{\mathrm{dc}} \leqslant 1.0 \mathrm{~T}$ and the corresponding data are plotted in figures $6(\mathrm{a})$ and (b). It is observed that at $H_{\mathrm{dc}}=0.5 \mathrm{~T}$ as well as $H_{\mathrm{dc}}=1.0 \mathrm{~T}$ the MR becomes significant around $T_{\mathrm{C}}$, while the peak in the MR occurs at a lower temperature. This feature, as also pointed out by de Andres et al [36], is in sharp contrast to the high-field MR that peaks around $T_{\mathrm{C}}$. For the LCMO thin film grown on STO the low-field MR becomes significant at $T_{\mathrm{C}}$, while the peak MR of $\sim 7 \%$ and $18 \%$ respectively at $H_{\mathrm{dc}}=0.5$ and $1.0 \mathrm{~T}$ occurs at a lower temperature around $T \sim 220 \mathrm{~K}$. The same behaviour is observed in the case of films on LAO and $\mathrm{MgO}$ although the MR values in this case are more than double the corresponding values measured for LCMO film on STO. However, apart from the magnitude, the temperature spread of MR around the respective peak MR values also increases as one moves from a film on STO (low lattice relaxation) to a film on $\mathrm{MgO}$ (high lattice relaxation). The LCMO film on STO by virtue of low lattice relaxation $(\sim 0.4 \%)$ is expected to have a relatively smaller density of extrinsic defects, while the films on LAO (lattice relaxation $\sim 3.06 \%$ ) and $\mathrm{MgO}$ (lattice relaxation $\sim 6.411 \%$ ) have much a higher 

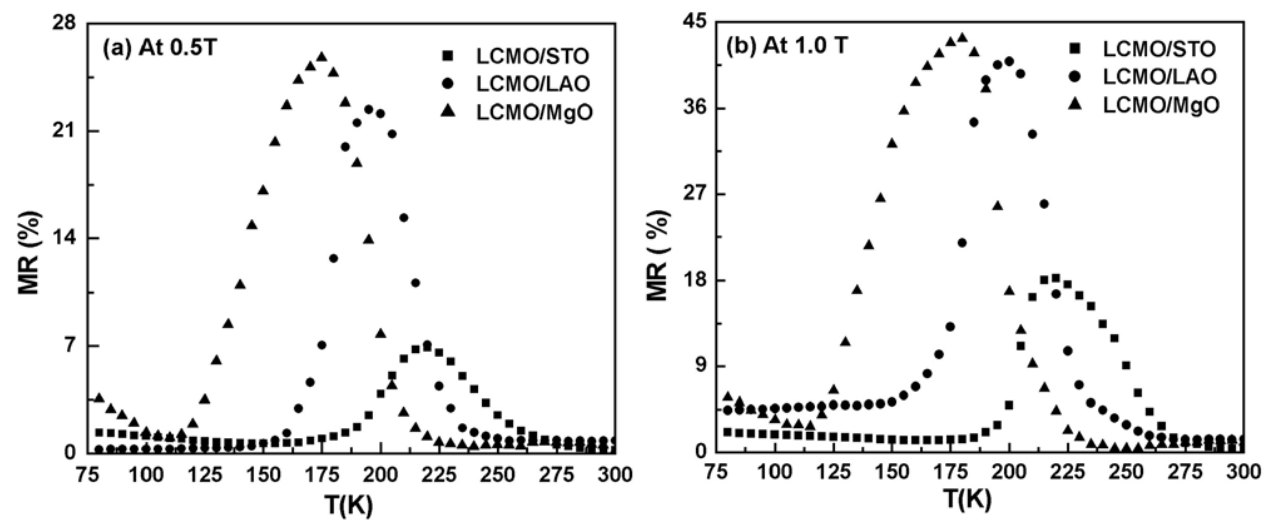

Figure 6. The temperature dependence of MR for LCMO films in an applied magnetic field of (a) $0.5 \mathrm{~T}$ and (b) $1.0 \mathrm{~T}$.

density of disorders and defects. It has been discussed by de Andres et al [36] that in epitaxial thin films the low-field MR around $T_{\mathrm{C}}$ or $T_{\mathrm{IM}}$ is related to the lattice distortions that could result as a consequence of the lattice relaxation-induced defects such as stacking faults. In the present investigation it has been shown from the rocking curve analysis as well as the temperature dependence of resistivity in the paramagnetic state that lattice distortions indeed increase with increasing lattice relaxation. It is known that around $T_{\mathrm{C}}$ polaron clusters form in the FM phase of the $30 \%$ doped LCMO. This polaron cluster formation is due to a drastic reduction in the bandwidth due to the lattice distortions around the defects. Since in the present investigation the density of defects increases as the lattice relaxation increases, the polaronic density is also expected to increase as one moves from STO to LAO and then to $\mathrm{MgO}$. These polarons are weakly bound in the FM phase and consequently can be delocalized by a relatively weak magnetic field. Thus the low-field MR in epitaxial thin films around $T_{\mathrm{C}}$ or $T_{\mathrm{IM}}$ has its origin in the delocalization of the small polaron clusters formed around $T_{\mathrm{C}}$ or $T_{\mathrm{IM}}$ in the FM phase.

The contribution of the defects/disorders is more or less confined to the temperature regime around $T_{\mathrm{C}}$ and $T_{\mathrm{IM}}$. In the LCMO films, polaron clusters form in the ferromagnetic phase around $T_{\mathrm{C}}$ by a drastic reduction of the bandwidth due to the lattice distortions around the defects. The decrease in the bandwidth caused by lattice distortion is given by the NarimanovVarma [37] formula

$$
W=W_{0} \cos \frac{\theta}{2} \exp \left(-\frac{k(\delta u)^{2}}{\hbar \omega_{0}}\right) .
$$

Here $\theta$ is the angle between the two Mn spins and $\delta u$ are lattice distortions that can be dynamic (e.g. phonons), intrinsic (related to La substitution by a divalent cation) or due to lattice defects around impurities. The lattice distortions around the defects favour the localization of polarons ( $T_{0}$ increases). As proposed by de Andres et al [36] these polarons are rather weakly bound in the ferromagnetic phase and therefore can be delocalized by a relatively smaller dc magnetic field. These small polarons can only be formed near $T_{\mathrm{C}}$ when the carrier bandwidth is reduced either by spin disorder or by lattice fluctuations. In the present case this is quite obvious from the decrease in the $T_{\mathrm{IM}}$ values with increasing lattice relaxation and also from the fact that the value of the parameter $T_{0}$ that eventually determines that localization length increases as the magnitude of the lattice relaxation increases in the LCMO films on STO, LAO and MgO. 


\section{Conclusions}

We have synthesized epitaxial $\mathrm{La}_{0.7} \mathrm{Ca}_{0.3} \mathrm{MnO}_{3}$ films on $\mathrm{LaAlO}_{3}, \mathrm{SrTiO}_{3}$ and $\mathrm{MgO}$ substrates and investigated the effect of strain relaxation on the magnetotransport properties of these films. It has been found that all the films are epitaxial, single phase and have a cubic unit cell. The outof-plane lattice parameter are 3.878, 3.889 and 3.899 Å respectively for films on STO, LAO and $\mathrm{MgO}$. The FWHM as evaluated through rocking curves ( $\omega$ scan) turns out to be $0.39^{\circ}, 0.52^{\circ}$ and $1.24^{\circ}$ for films on STO, LAO and MgO respectively. As the films investigated in the present case are thicker $(\sim 200 \mathrm{~nm})$, the substrate-induced strain does not persist but it relaxes, and the relaxation gives rise to extrinsic distortions/defects such as dislocations, grain-boundaries, stacking faults, and cationic vacancies. The lattice relaxation has been found to be $0.384 \%$, $3.057 \%$ and $6.411 \%$ for films deposited on STO, LAO and $\mathrm{MgO}$. The observed $T_{\mathrm{C}}$ values are $\sim 244,218$ and $186 \mathrm{~K}$ respectively for LCMO films deposited on STO, LAO and MgO. The $T_{\mathrm{IM}}$ of LCMO films on STO, LAO and MgO is found to be $\sim 243,217$ and $191 \mathrm{~K}$. The decrease in $T_{\mathrm{IM}}$ and $T_{\mathrm{C}}$ for $\mathrm{MgO}$ films has been explained on the basis of lattice strain relaxation. A higher degree of relaxation creates more defects which affect the magnetotransport properties and $T_{\mathrm{IM}}\left(T_{\mathrm{C}}\right)$ decreases. Keeping in view the variable presence of disorder in the present films, we have analysed the transport above $T_{\mathrm{C}}$ through Mott's VRH model. Based on this model the increase in lattice relaxation will produce defects, which will result in decrease of the tendency of charge localization (the hopping distance also decreases) and so will $T_{\mathrm{IM}}$ and $T_{\mathrm{C}}$ decrease. As already described in section 2.4.3 these are in keeping with our experimental results. A detailed study on lattice strain relaxation by employing cross-sectional TEM and AFM is in progress and results will be forthcoming.

\section{Acknowledgments}

This work was supported financially by UGC and CSIR, New Delhi. One of the authors (PKS) acknowledges CSIR, New Delhi for the award of research fellowships. The authors are also grateful to Professors A R Verma, C N R Rao, T V Ramakrishnan, Vikram Kumar, S B Ogale, A K Raychaudhari, Dr Kishan Lal and Dr N Khare for valuable discussions. We also thank Dr Alok Banarjee for helping in magnetization measurements.

\section{References}

[1] von Helmholt R, Wecker J, Holzapfel B, Schultz L and Samwer K 1993 Phys. Rev. Lett. 712331

[2] Jin S, Tiefel T H, McCormack M, Fastnacht R A, Ramesh R and Chen L H 1994 Science 264413

[3] Ju H L, Kwon C, Li Q, Greene R L and Venkatesan T 1994 Appl. Phys. Lett. 652108

[4] Jin S, Tiefel T H, McCormack M, O’Bryan H M, Chen L H, Ramesh R and Schurig D 1995 Appl. Phys. Lett. 67557

[5] Prellier W, Rajeswari M, Venkatesan T and Greene R L 1999 Appl. Phys. Lett. 751446

[6] Koo T Y, Park S H, Lee K B and Jeong Y H 1997 Appl. Phys. Lett. 71977

[7] Praus R B, Leibold B, Gross G M and Habermeier H U 1999 Appl. Surf. Sci. 13840

[8] Kwon C, Robson M C, Kim K C, Gu J Y, Lofland S E, Bhagat S M, Trajanovic Z, Rajeswari M, Venkatesan T, Kratz A R, Gomez R D and Ramesh R 1997 J. Magn. Magn. Mater. 172229

[9] Razavi F S, Gross G, Habermeier H U, Lebedev O, Amelinckx S, Van Tendeloo G and Vigliante A 2000 Appl. Phys. Lett. 76155

[10] Khartsev S I, Johnsson P and Grishin A M 2000 J. Appl. Phys. 872394

[11] Izumi M, Konishi Y, Nishihara T, Hayashi S, Shinohara M, Kawasaki M and Tokura Y 1998 Appl. Phys. Lett. 732497

[12] Dho J, Hur N H, Kim I S and Park Y K 2003 J. Appl. Phys. 947670

[13] Rao R A, Lavric D, Nath T K, Eom C B, Wu L and Tsui F 1999 J. Appl. Phys. 854794 
[14] Shreekala R, Rajeswari M, Srivastava R C, Ghosh K, Goyal A, Srinivasu V V, Lofland S E, Bhagat S M, Downes M, Sharma R P, Ogale S B, Greene R L, Ramesh R, Venkatesan T, Rao R A and Eom C B 1999 Appl. Phys. Lett. 741886

[15] Millis A J, Littlewood P B and Shraiman B I 1995 Phys. Rev. Lett. 745144

[16] Robson M C, Kwon C, Lofland S E, Ogale S B, Bhagat S M, Rajeswari M, Venkatesan T and Ramesh R 2000 J. Electroceram. 4167

[17] Bibes M, Balcells L I, Valencia S, Fontcuberta J, Wojcik M, Jedryka E and Nadolski S 2001 Phys. Rev. Lett. 87067210

[18] Zhang J, Tanaka H, Kanki T, Choi J H and Kawai T 2001 Phys. Rev. B 64184404

[19] Kanki T, Tanaka H and Kawai T 2001 Phys. Rev. B 64224418

[20] Kanki T, Tanaka H and Kawai T 2004 Phys. Rev. B 70125109

[21] Gommert E, Cerva H, Wecker J and Samwer K 1999 J. Appl. Phys. 855417

[22] Prellier W, Lecoeur Ph and Mercey B 2001 J. Phys.: Condens. Matter 13 R915

[23] Haghiri-Gosnet A M and Renard J P 2003 J. Phys. D: Appl. Phys. 36 R127

[24] Gong G Q, Gupta A, Xiao G, Lecoeur Ph and McGuire T R 1996 Phys. Rev. B 54 R3742

[25] Prellier W, Haghiri-Gosnet A M, Mercey B, Lecoeur Ph, Hervieu M, Simon Ch and Raveau B 2000 Appl. Phys. Lett. 771023

[26] Mills A J, Darling T and Migliori A 1998 J. Appl. Phys. 831588

[27] Pradhan A K, Sahu D R, Roul B K and Feng Y 2002 Appl. Phys. Lett. 813597

[28] Murugavel P, Lee J H, Yoon J G, Noh T W, Chung J S, Heu M and Yoon S 2003 Appl. Phys. Lett. 821908

[29] Klein J, Philipp J B, Reisinger D, Opel M, Marx A, Erb A, Alff L and Gross R 2003 J. Appl. Phys. 937373

[30] Yeh N C, Vasquez R P, Beam D A, Fu C C, Huynh J and Beach G 1997 J. Phys.: Condens. Matter 93713

[31] Mott N F 1979 Metal-Insulator Transitions (London: Taylor and Francis)

[32] Viret M, Ranno L and Coey J M D 1997 Phys. Rev. B 558067

[33] Ziese M and Srinitiwarawong C 1998 Phys. Rev. B 5811519

[34] Ravindranath V, Ramachandra Rao M S, Rangarajan G, Lu Y, Klein J, Klingeler R, Uhlenbruck S, Büchner B and Gross R 2001 Phys. Rev. B 63184434

[35] Okimoto Y, Katsufuji T, Ishikawa T, Urushibara A, Arima T and Tokura Y 1995 Phys. Rev. Lett. 75109

[36] de Andres A, Taboda S, Colino J M, Rameriz R, Garcia-Hernandez M and Marteniz J L 2002 Appl. Phys. Lett. 81319

[37] Narimanov E E and Varma C M 2001 Preprint cond-mat/0110047 